Tohoku J. Exp. Med., 2008, 215, 355-361

\title{
Gentle Exercise of 40 Minutes with Dietary Counseling is Effective in Treating Metabolic Syndrome
}

\author{
Takahiro Mitsui, ${ }^{1}$ Kiyoshi Shimaoka, ${ }^{2}$ Shigeki Tsuzuku, ${ }^{3}$ Taeko Kajioka ${ }^{4}$ and \\ HisATAKa SAKaKIBARA ${ }^{5}$ \\ ${ }^{1}$ Faculty of Human Health, Hachinohe University, Hachinohe, Japan \\ ${ }^{2}$ Research Center of Health, Physical Fitness and Sports, Nagoya University, Nagoya, Japan \\ ${ }^{3}$ Department of Public Health, Kochi Medical School, Kochi, Japan \\ ${ }^{4}$ School of Public Health, Graduate School of Medicine, The University of Tokyo, Tokyo, Japan \\ ${ }^{5}$ Department of Health Sciences, School of Medicine, Nagoya University, Nagoya, Japan
}

\begin{abstract}
Obese people may succeed in reducing their weight temporarily, but most of them regain the lost weight within a few years. We, therefore, recommend moderate long-term changes in habit rather than a strict temporary program. The purpose of this study was to examine whether physical activity of 40 minutes, mainly walking, was effective in improving or preventing metabolic syndrome when combined with dieting. Participants, all of whom had a waist circumference $\geq 85 \mathrm{~cm}$ (men) or $\geq 90 \mathrm{~cm}$ (women), were assigned into one of two groups: the control group (10 men and 11 women, age: $64.2 \pm 3.4$ [SD] years) and the intervention group (10 men and 12 women, age: $62.4 \pm 4.2$ years). Participants $(n=22)$ enrolled in a 52-week program; their diets were assessed using a 3-day dietary record and daily steps were measured with a pedometer. No significant change was seen in their dietary records, but the mean number of daily steps increased from 5,806 to 8,000-9,000. In the intervention group, BMI, waist circumference, and systolic and diastolic blood pressures decreased significantly by week 12 (all $p<0.001$ ) and remained low until week 52 . In fact, no significant change was seen between weeks 12 and 52. The control group showed no significant changes in all variables. These results indicate that gentle exercise of about 40 minutes in combination with dieting is effective in preventing metabolic syndrome among a sedentary population, although its effect may be limited.

metabolic syndrome; self-training program; pedometer.
\end{abstract}

Tohoku J. Exp. Med., 2008, 215 (4), 355-361.

(C) 2008 Tohoku University Medical Press

Obesity, especially the accumulation of visceral adipose tissue, is linked to a cluster of metabolic and vascular disorders that have collectively been termed metabolic syndrome (Reaven 1988). In Japan, metabolic syndrome is defined as having a waist circumference $\geq 85$ for men or $\geq 90 \mathrm{~cm}$ for women, plus two or more of the following abnormalities: systolic blood pressure $(\mathrm{SBP}) \geq$ $130 \mathrm{mmHg}$ or diastolic blood pressure (DBP) $\geq$ $85 \mathrm{mmHg}$, blood glucose $\geq 110 \mathrm{mg} / \mathrm{dL}$, high density lipoprotein (HDL) $\leq 40 \mathrm{mg} / \mathrm{dL}$, or triacylglycerol $\geq 150 \mathrm{mg} / \mathrm{dL}$ (Matsuzawa 2005). Therefore, reduction of body fat and of waist circumference, which is a simple indicator of the

Received April 8, 2008; revision accepted for publication July 4, 2008.

Correspondence: Kiyoshi Shimaoka, Research Center of Health, Physical Fitness and Sports, Nagoya Univer-

sity, Furocho, Chikusaku, Nagoya 464-8601, Japan.

e-mail: kshima@htc.nagoya-u.ac.jp 
amount of visceral adipose tissue (Pouliot et al. 1994), is indispensable for the prevention and treatment of metabolic syndrome.

The most effective way to reduce body fat is to combine an adequate diet with exercise. Reducing body mass by diet alone can result in the loss of fat-free mass (FFM) and a reduction in resting metabolism rate (RMR) (Ballor et al. 1988; Ross et al. 1995). In addition, intense dieting may result in temporary weight reduction, but in most cases the lost weight is regained within several years (Johnson and Drenick 1977). To achieve long-term weight loss, it is essential to sustain both a moderate diet and exercise over many years.

Authorities recommend that individuals engage in 30 minutes of moderate-intensity physical activity, such as brisk walking, on most days of the week, preferably every day (US Department of Health and Human Services 1996). Traditionally, aerobic forms of exercise such as walking, cycling, and swimming have been preferred for middle-aged and older people. Today, resistance training is recommended as well because it is believed to reverse or at least slow the loss of muscle function due to advancing age (American Diabetes Association 2000).

Successful diet programs previously reported in academic papers are generally supervised, intense, and require a long period of training time. A simple and voluntary program is preferable, as it is more likely to result in sustained long-term weight control. The purpose of this study was to determine whether walking and self-weight resistance training, i.e. weight training using the body's own weight rather than equipment such as dumbbells or barbells, in combination with a dietary counseling program, is effective in the prevention or improvement of metabolic syndrome and sustainable without a trainer's supervision.

\section{Methods}

\section{Participants}

All participants were Yokkaichi City residents who responded to a public announcement. Most of them underwent a physical health examination conducted by the city. Subjects who met the following criteria were included in this study: 50 to 69 years of age, waist circumference $\geq 85 \mathrm{~cm}$ (men) or $\geq 90 \mathrm{~cm}$ (women), no regular exercise for the past 6 months, present non-smoker, ambulant, no history of serious disease such as diabetes, cancer, stroke, heart disease, or kidney disease requiring dialysis. The female participants were all postmenopausal. Forty-six persons (21 men and 25 women) met these criteria; 24 of them (11 men and 13 women) were randomly assigned to the intervention group, and $22(10$ men and 12 women) to the control group. The participants were all apparently healthy, but some were taking medication for the following syndromes: hypertension ( 8 participants); knee or hip pain (6); osteoporosis (3), gout (3), and gastritis (3). We asked participants not to make any changes in their use of medications during the study period, insofar as this was possible. Written informed consent was obtained from all participants, and our program was approved by the Research Ethics Committee of the Yokkaichi City Office.

\section{Exercise and nutrition intervention}

Briefly, our program consisted of 52 weeks of selfdirected training and dietary counseling. In order to become accustomed to the diet and training, the intervention group attended lectures at a city gymnasium once a week until week 12 , once every other week between week 13 and week 26, and thereafter once a month. They were lectured on nutrition, cooking, exercise, and preventive medicine.

Their training consisted of walking of 20-30 minutes and 2 or 3 self-weight resistance exercises such as squats, sit-ups, push-ups with knees on the floor, and hamstring stretches, each of which they performed for 10 minutes. Although we predetermined the length of time spent on each exercise, the number of repetitions and the intensity of these exercises were determined by individual participants. Time was always provided for warm-up and cool-down periods. The total training time lasted up to 40 minutes.

Participants were advised to perform self-training for 30-40 minutes a day, initially two or three times per week; when they became used to this level of training, they were asked to increase the number of days on which they exercised to more than 5 per week. Their walking volume was measured in steps/day using a pedometer (Yamasa EM-180, Tokyo, Japan). Each participant was given a pedometer and asked to record his or her daily steps, from waking until going to bed, excluding water 
activities. The mean number of steps/day was calculated for each week. Participants in the intervention group submitted their records of exercise each time they came to the gymnasium for a lecture. The control group sent their records in by mail.

Before the program began, we collected information on the dietary intake of all participants using a 3-day dietary record. The dietary assessment was based on the Standard Tables of Food Composition in Japan (5th ed.). Based on the results of this assessment, a trained dietician counseled each participant in the intervention group on his or her dietary habits according to the Dietary Reference Intakes for Japanese, 2005. Although total calories, carbohydrates, and fat were not restricted, the intervention group was advised to refrain from eating snacks and from eating too much dessert, including fruit.

Measurements of anthropometrics, blood pressure, and blood constituents

These assessments were conducted at the start of the program, at week 12, and at week 52. All measurements were conducted after overnight fasting. Body mass index (BMI) was calculated by dividing weight $(\mathrm{kg})$ by height $\left(\mathrm{m}^{2}\right)$. Waist circumference was measured at the umbilical level, with the participant standing erect and the abdomen relaxed. Blood pressure was measured using a mercury sphygmomanometer. The blood samples were drawn from the medial cubital vein, and biochemical measurements were conducted in a routine manner at a certified laboratory (MITSUBISHI CHEMICAL
MEDIENCE, Yokkaichi, Japan). Serum total cholesterol (T-C), high-density lipoprotein cholesterol, and triacylglycerol concentrations were assayed using enzymatic methods, and serum glucose concentration was measured according to the glucose oxidase method.

\section{Data analysis}

Data are expressed as mean \pm S.D. When the data were distributed abnormally, the median (range) was adopted. Variables between the groups and time-course changes within a group were analyzed with a two-way ANOVA. In the presence of a significant $F$ value, Ryan's method was used to compare within each group. When an interaction between variables and time was identified, we used an unpaired $t$-test at each time point. All statistical analyses were performed using ANOVA4 (Kiriki, 2002). $p<0.05$ was considered to be statistically significant.

\section{Results}

Two participants in the intervention group and 1 in the control group dropped out of the program after week 12 for personal reasons. We were left with 22 participants $(10$ men and 12 women) in the intervention group and 21 (10 men and 11 women) in the control group for statistical analysis. Five subjects in the intervention group and 7 in the control group had actually been diagnosed with metabolic syndrome. There were no

TABLE 1. Baseline characteristics of the participants.

\begin{tabular}{lcc}
\hline & Control group & Intervention group \\
\hline Age, years & $64.2 \pm 3.4$ & $62.4 \pm 4.2$ \\
Height, cm & $161.8 \pm 8.4$ & $160.6 \pm 10.0$ \\
Weight, $\mathrm{kg}$ & $67.4 \pm 10.6$ & $64.0 \pm 8.9$ \\
BMI, $\mathrm{kg} / \mathrm{m}^{2}$ & $25.6 \pm 2.5$ & $24.8 \pm 2.2$ \\
Waist circumference, cm & $94.9 \pm 6.2$ & $92.7 \pm 5.1$ \\
SBP, mmHg & $129 \pm 12$ & $139 \pm 22$ \\
DBP, mmHg & $78 \pm 11$ & $81 \pm 13$ \\
Blood glucose, mg/dL & $98 \pm 16$ & $96 \pm 12$ \\
T-C, mg/dL & $231 \pm 24$ & $225 \pm 34$ \\
HDL-C, mg/dL & $51 \pm 12$ & $52 \pm 9$ \\
Triacylglycerol, $\mathrm{mg} / \mathrm{dL}$ & $146(25-326)$ & $120(57-232)$
\end{tabular}

Data are expressed as mean \pm S.D. or median (range).

BMI; body mass index; SBP, systolic blood pressure; DBP, diastolic blood pressure; T-C, total cholesterol; HDL-C, high-density lipoprotein cholesterol. 
T. Mitsui et al.

TABLE 2. Anthropometry, blood pressure, blood constituents and changes during the study period.

\begin{tabular}{lcccrrr}
\hline & W0 & W12 & W52 & $\Delta$ W12-W0 & \multicolumn{1}{c}{ W52-W0 } & $\Delta$ W52-W12 \\
\hline Control group & & & & & & \\
BMI, kg/m & & & & & \\
Waist circumference, cm & $94.9 \pm 2.5$ & $25.5 \pm 2.6$ & $25.5 \pm 2.6$ & -0.1 & -0.1 & 0 \\
SBP, mmHg & $129.0 \pm 12.4$ & $128.0 \pm 13.7$ & $127.8 \pm 13.6$ & -1.0 & -1.2 & -0.2 \\
DBP, mmHg & $78.1 \pm 11.1$ & $76.5 \pm 9.6$ & $75.7 \pm 10.9$ & -1.6 & -2.4 & -0.8 \\
Blood glucose, mg/dL & $97.6 \pm 15.7$ & $96.4 \pm 10.2$ & $98.5 \pm 12.7$ & -1.2 & 2.1 & 0.9 \\
T-C, mg/dL & $230.9 \pm 23.8$ & $225.9 \pm 30.7$ & $236.8 \pm 30.3$ & -5.0 & -5.9 & 0.9 \\
HDL-C, mg/dL & $50.7 \pm 12.1$ & $52.6 \pm 11.1$ & $52.0 \pm 11.8$ & 1.9 & 1.3 & -0.6 \\
Triacylglycerol, mg/dL & $146(25-326)$ & $138(72-274)$ & $155(69-392)$ & -8.0 & -9.0 & 17.0 \\
\hline Intervention group & & & & & & \\
BMI, kg/m & & & & & \\
Waist circumference, cm & $92.8 \pm 2.2$ & $24.0 \pm 2.2^{*}$ & $23.7 \pm 2.4 *$ & -0.8 & -1.1 & -0.3 \\
SBP, mmHg & $139.3 \pm 22.2$ & $130.7 \pm 19.3^{*}$ & $129.3 \pm 17.5$ & -8.6 & -10.0 & -1.4 \\
DBP, mmHg & $81.4 \pm 13.0$ & $75.9 \pm 12.2^{*}$ & $74.7 \pm 11.5^{*}$ & -5.5 & -6.7 & -1.2 \\
Blood glucose, mg/dL & $96.3 \pm 12.4$ & $92.6 \pm 10.7$ & $91.1 \pm 11.8$ & -3.7 & -5.2 & -1.5 \\
T-C, mg/dL & $225.4 \pm 34.0$ & $215.5 \pm 26.8$ & $220.6 \pm 30.9$ & -9.9 & -4.8 & 5.1 \\
HDL-C, mg/dL & $51.6 \pm 9.2$ & $51.8 \pm 12.1$ & $54.4 \pm 11.9$ & 0.2 & 2.8 & 2.6 \\
Triacylglycerol, mg/dL & $120(57-232)$ & $100(54-249)^{\#}$ & $112.5(48-316)$ & -20 & -7.5 & 12.5 \\
\hline
\end{tabular}

Data are expressed as mean \pm S.D. or median (range).

${ }^{*} p<0.001$ vs. W0, ${ }^{\sharp} p<0.05$ vs. control group.

W12, week 12; W52: week 52 .

significant differences between the two groups in any of the baseline variables at the start of the study (Table 1) or in changes to dietary intake during the course of the study (Table 3 ).

Among the participants in the intervention group, the number of steps per day gradually increased, starting around week 4, from a baseline mean of 5,806 to a mean ranging from 8,000 to 9,000 (Fig. 1). Most of these participants conducted several self-weight exercises as well. Among the control group, none of the anthropometric or biochemical variables changed significantly, but the intervention group showed significant changes: BMI $(p<0.0001)$, waist circumference $(p<0.0001), \operatorname{SBP}(p<0.001)$, and DBP $(p<0.001)$ were all significantly reduced by week 12, and remained lower at week 52. There were, however, no significant changes in these variables in the intervention group between week 12 and week 52. The blood constituents of the participants in the intervention group did not change significantly.

Between the two groups, there were significant differences in waist circumference at week $12(p=0.0068)$ and at week $52(p=0.0071)$, and in triacylglycerols at week $12(p=0.013)$. No other variables differed significantly between the groups at week 12 or week 52 .

\section{Discussion}

Strict dietary restriction and intense exercise are not suitable for successful long-term weight control, and they often pose a risk of damage to health. Furthermore, it remains to be determined whether programs conducted under supervised or hospitalized conditions are as effective in the long-term as self-conducted programs. Therefore, we assume that a moderate diet and simple selfdirected exercise are preferable for weight control and the prevention of lifestyle-related disease. 
TABLE 3. Estimated daily intake assessed using a 3-day food record.

\begin{tabular}{cccc}
\hline & Baseline & W12 & W52 \\
\hline Control group & & & \\
Total energy, kcal/day & $2,044 \pm 481$ & $2,098 \pm 467$ & $1,983 \pm 547$ \\
Protein, g/day & $72.1 \pm 16.4$ & $72.3 \pm 23.6$ & $71.1 \pm 16.7$ \\
Fat, g/day & $56.7 \pm 20.2$ & $57.4 \pm 27.2$ & $49.1 \pm 17.9$ \\
Carbohydrate, g/d & $292.8 \pm 78.2$ & $283.2 \pm 71.4$ & $296.1 \pm 89.1$ \\
Dietary fiber, g/d & $15.3 \pm 4.5$ & $16.3 \pm 5.1$ & $16.9 \pm 5.7$ \\
Calcium, mg/day & $565 \pm 245$ & $610 \pm 267$ & $584 \pm 238$ \\
\hline Intervention group & & & \\
Total energy, kcal/day & $2,074 \pm 664$ & $2,083 \pm 548$ & $2,074 \pm 627$ \\
Protein, g/day & $83.4 \pm 23.4$ & $81.5 \pm 25.7$ & $78.0 \pm 17.1$ \\
Fat, g/day & $48.4 \pm 24.0$ & $51.5 \pm 19.6$ & $49.8 \pm 19.4$ \\
Carbohydrate, g/d & $311.1 \pm 100.9$ & $302.5 \pm 83.1$ & $313.0 \pm 111.4$ \\
Dietary fiber, g/d & $18.5 \pm 7.3$ & $18.4 \pm 6.1$ & $19.2 \pm 5.3$ \\
Calcium, mg/day & $693 \pm 338$ & $697 \pm 236$ & $701 \pm 285$ \\
\hline
\end{tabular}

Data are expressed as the mean \pm S.D.

W12, week 12; W52; week 52.

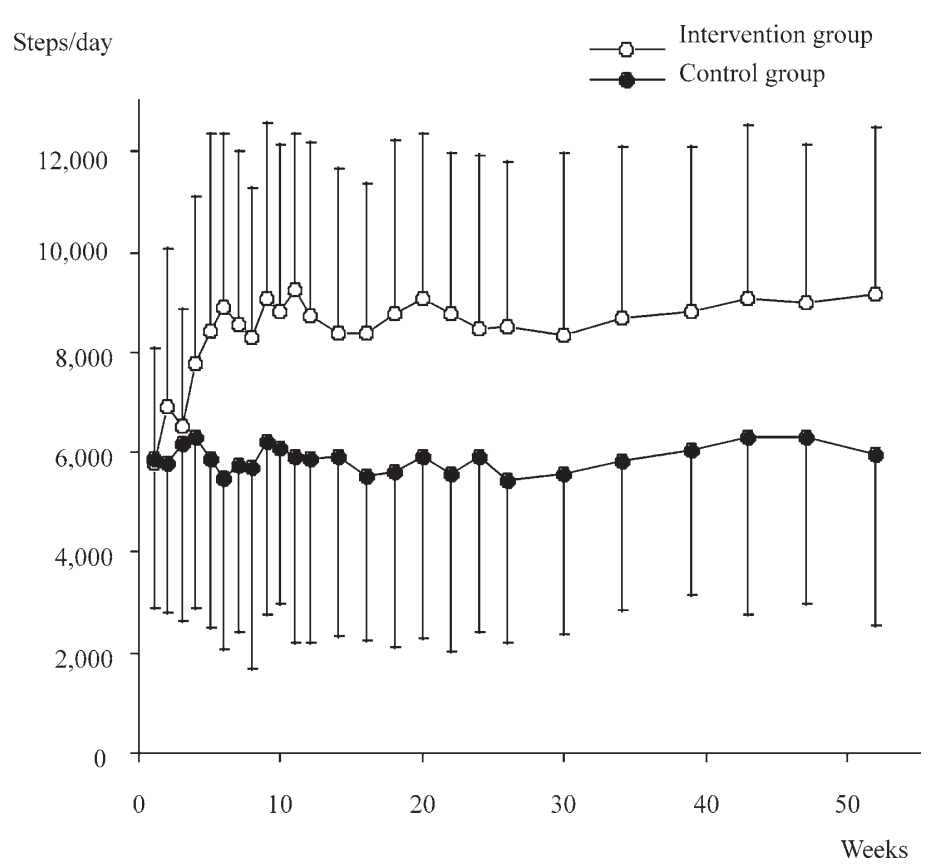

Fig. 1. Mean steps/day during the study period. Vertical bars represent the standard deviation.

Training intervention studies usually adopt a single type of exercise, either endurance or resistance training, probably because this tends to make study design more clear and data analysis easier. Yet people who are engaged in sports rath- er than participating in a study usually conduct various types of exercise, including stretching and resistance training to prevent injuries and improve body performance. Walking was the primary exercise and self-weight resistance training was 
secondary in this program. Walking is the most accessible form of exercise for all age groups, and, when combined with light resistance training, it seems to be effective in preventing muscle deterioration due to aging.

Another advantage of using walking as the primary form of exercise in this study is the relative ease with which it can be measured. Strict measurement of the quantity of other forms of exercise can be difficult, but walking can be objectively and reliably measured by recording the number of steps taken per day. Our participants increased their number of daily steps by approximately 2,500-3,000, which indicates that they did conduct 30 to 40 minutes of daily exercise as instructed during the study period.

The practice of recording steps per day with a pedometer has recently gained in popularity. Japanese walking clubs and pedometer manufacturers have been recommending 10,000 steps/day for more than 40 years, because walking 10,000 steps/day results in the expenditure of approximately $300 \mathrm{kcal} /$ day, although this figure varies with body size and walking speed (Hatano 1993). For the same reason, 10,000 steps/day is often set as a target value in research papers (Tudor-Locke and Bassett 2004). A goal of 10,000 steps/day seems to be reasonable for people, who may already be walking 6-7,000 steps/day, but it may be rather high for inactive people, who usually walk $<5,000$ steps/day. There is evidence that only $38-50 \%$ of women reach 10,000 steps/day on any given day, even with a prescribed 30-minute walk (Wilde et al. 2001). We know that a total of 10,000 steps/day may not be a realistic goal for everyone, but we believe that an increase of approximately 3,000 steps/day is practical even for inactive people. The low drop-out ratio in this study $(8.3 \%)$ and the fact that participants were able to sustain their increased number of daily steps for nearly one year indicate that most of the participants in the present study found our program feasible, and became accustomed to it.

We observed improvements in waist circumference, BMI, and blood pressures; this is in sharp contrast to the results obtained by Swartz et al. (2003) and Tudor-Locke et al. (2004), who reported that a 30-minute daily walk did not result in reduced BMI or waist circumference in obese people with a BMI $>30 \mathrm{~kg} / \mathrm{m}^{2}$. Their participants used pedometers and reported an increase of 3,000 steps/day on average. The most probable reason why the participants in these other studies failed to reduce body fat while our subjects succeeded is that the other studies did not provide dietary intervention. Thirty minutes of moderate activity results in the expenditure of approximately 150 kcal (Tudor-Locke and Bassett Jr. 2004), an amount of energy which may be insufficient to reduce body weight without dieting. Furthermore, moderate physical activity stimulates increased energy intake, which results in unchanged body weight (Melzer et al. 2005). We did not find any significant reduction in energy intake, even in the intervention group; since we did not strictly instruct them to change their dietary habits, any changes they did make may have been too small to detect with our method of dietary assessment.

SBP and DBP were significantly reduced in the intervention group, which is in agreement with findings in previous walking program studies (Moreau et al. 2001; Swartz et al. 2003; Tully et al. 2005). In blood constituents, on the other hand, no significant changes were observed, probably because the participants' baseline values were already within the normal ranges. In general, both endurance exercise and resistance exercise have a beneficial effect on these indices, but the magnitude of this effect is dependent on the baseline value.

It is worth noting that there was no significant change in BMI or waist circumference between week 12 and week 52. This result may indicate a limitation of this program. Many factors such as dietary intake, energy expenditure, social status, and genetics are implicated in changes in body weight, but energy balance is the determining factor. It appears that our participants' increased energy expenditure was balanced out by increased energy intake after 12 weeks. The participants were rather overweight compared to the Japanese population at large, but their average BMI was within the normal range: $24.7 \pm 2.1$ $\mathrm{kg} / \mathrm{m}^{2}$ at the start of the study and $24.0 \pm 2.2$ 
$\mathrm{kg} / \mathrm{m}^{2}$ at week 12 , which means that most of the participants did not need to substantially reduce their body weight.

Today, obesity is a public health problem throughout the world. A number of methods involving diet, exercise, and lifestyle changes have been proposed to resolve it. Treatment such as surgery, medication, or hospitalization may be necessary for extremely obese people, but moderate changes in dietary and exercise habits are reasonable first steps for most obese people. It is certain that insufficient physical activity and overeating, particularly eating too much junk-foods and foods that are high in fat, are causes of obesity. We suggest that the best way to solve the problem of obesity is to compensate for its causes.

\section{Acknowledgments}

The authors would like to thank the public nurses and dieticians of Yokkaichi City for technical assistance.

\section{References}

American Diabetes Association: Diabetes mellitus and exercise (Position Statement). (2000) Diabetes Care, 23, Suppl. 1, S50-S54.

Ballor, D.L., Katch, V.L., Becque, M.D. \& Marks, C.R. (1988) Resistance weight training during caloric restriction enhances lean body weight maintenance. Am. J. Clin. Nutr., 47, 19-25.

Hatano, Y. (1993) Use of the pedometer for promoting daily walking exercise. ICHPER, 29, 4-8.

Johnson, D. \& Drenick, E.J. (1977) Therapeutic fasting in morbid obesity. Arch. Intern. Med., 137, 1381-1382.

Kiriki K (2002) ANOVA4 on the Web. http://www.hju.ac.jp/ kiriki/anova4/about.html

Kusuoka, H. \& Hoffman, J.I. (2002) Advice on statistical analysis for Circulation Research. Circ. Res., 91, 662-671.
Matsuzawa, Y. (2005) Metabolic syndrome--definition and diagnostic criteria in Japan. J. Atheroscler. Thromb., 12, 301.

Melzer, K., Kayser, B., Saris, W.H. \& Pichard C. (2005) Effects of physical activity on food intake. Clin. Nutr., 24, 885-895.

Moreau, K.L., Degarmo, R., Langley, J., McMahon, C., Howley, E.T., Bassett, D.R. Jr. \& Thompson, D.L. (2001) Increasing daily walking lowers blood pressure in postmenopausal women. Med. Sci. Sports Exerc., 33, 1825 1831.

Pouliot, M.C., Després, J.P., Lemieux, S., Moorjani, S., Bouchard, C., Tremblay, A., Nadeau, A. \& Lupien, P.J. (1994) Waist circumference and abdominal sagittal diameter: best simple anthropometric indexes of abdominal visceral adipose tissue accumulation and related cardiovascular risk in men and women. Am. J. Cardiol., 73, 460-468.

Ross, R., Pedwell, H. \& Rissanen, J. (1995) Response of total and regional lean tissue and skeletal muscle to a program of energy restriction and resistance exercise. Int. J. Obes. Relat. Metab. Disord., 19, 781-787.

Swartz, A.M., Strath, S.J., Bassett, D.R., Moore, J.B., Redwine, B.A., Groër, M. \& Thompson, D.L. (2003) Increasing daily walking improves glucose tolerance in overweight women. Prev. Med., 37, 356-362.

Tudor-Locke, C. \& Bassett, D.R. Jr. (2004) How many steps/ day are enough? Preliminary pedometer indices for public health. Sports Med., 34, 1-8.

Tudor-Locke, C., Bell, R.C., Myers, A.M., Harris, S.B., Ecclestone, N.A., Lauzon, N. \& Rodger, N.W. (2004) Controlled outcome evaluation of the First Step Program: a daily physical activity intervention for individuals with type II diabetes. Int. J. Obes. Relat. Metab. Disord., 28, 113-119.

Tully, M.A., Cupples, M.E., Chan, W.S., McGlade, K. \& Young, I.S. (2005) Brisk walking, fitness, and cardiovascular risk: a randomized controlled trial in primary care. Prev. Med., 41, 622-628.

Wilde, B.E., Sidman, C.L. \& Corbin, C.B. (2001) A 10,000 -step count as a physical activity target for sedentary women. Res. Q. Exerc. Sport, 72, 411-414.

US Department of Health and Human Services. Physical Activity and Health: a report of the Surgeon General. Atlanta: US Department of Health and Human Services, Center for Diseases Control and Prevention, National Center for Chronic Disease Prevention and Health Promotion, 1996. 